\title{
Detection of poxtA2, a Presumptive poxtA Ancestor, in a Plasmid from a Linezolid-Resistant Enterococcus gallinarum Isolate
}

\author{
(D) Ilaria Baccani, a Alberto Antonelli, ${ }^{\mathrm{a}, \mathrm{b}}$ Vincenzo Di Pilato, ${ }^{\mathrm{b}, \mathrm{c}}$ Marco Coppi, ${ }^{\mathrm{a}, \mathrm{b}}$ Tiziana Di Maggio, ${ }^{\mathrm{d}}$ Michele Spinicci, ${ }^{\mathrm{a}, \mathrm{e}}$ \\ Ana Liz Villagran, ${ }^{\mathrm{f}}$ Carmen Revollo, ${ }^{9}$ Alessandro Bartoloni, ${ }^{\mathrm{a}, \mathrm{e}} \mathbb{1}$ (Dian Maria Rossolinia,b \\ aDepartment of Experimental and Clinical Medicine, University of Florence, Florence, Italy \\ bMicrobiology and Virology Unit, Careggi University Hospital, Florence, Italy \\ ¿Department of Surgical Sciences and Integrated Diagnostics, University of Genoa, Genoa, Italy \\ dDepartment of Medical Biotechnologies, University of Siena, Siena, Italy \\ eInfectious and Tropical Diseases Unit, Careggi University Hospital, Florence, Italy \\ fHospital Básico Villa Montes, Villa Montes, Plurinational State of Bolivia \\ 9Instituto Nacional de Laboratorios de Salud "Dr. Nestor Morales Villazón" (INLASA), La Paz, Plurinational State of Bolivia
}

KEYWORDS poxtA, linezolid resistance, Enterococcus, IS1216

T he poxtA gene encodes a protein belonging to the $F$ lineage of the ATP-binding cassette superfamily, which can protect the bacterial ribosome from some antiribosomal antibiotics, including oxazolidinones (1). After the first identification in a methicillin-resistant Staphylococcus aureus of clinical origin (1), several reports of poxtA-positive isolates of Enterococcus spp. of animal and human origin from different countries have documented the broad and intersectoral dissemination of this transferable resistance gene, mainly among enterococci (2-8).

During a survey on antibiotic-resistant bacteria carried out in 2018 among the rural population living in the Bolivian Chaco region, an Enterococcus gallinarum (Eg-IV02) resistant to linezolid (MIC of $8 \mu \mathrm{g} / \mathrm{ml}$ by reference broth microdilution [9]) was isolated from a fecal swab collected from a healthy child after plating the swab on CNA-cv Sh medium (bioMérieux, Marcy l'Etoile, France) supplemented with $16 \mu \mathrm{g} / \mathrm{ml}$ of florfenicol.

Multiplex real-time PCR was positive for the poxtA gene but not for the optrA, cfr, or $c f r(B)$ genes, which are the other known transferable linezolid resistance determinants encountered in Gram-positive cocci.

Whole-genome sequencing (WGS) analysis of E. gallinarum Eg-IV02, performed using both Illumina MiSeq (Illumina, San Diego, CA) and MinION (Oxford Nanopore Technologies, Oxford, UK) platforms to yield a de novo hybrid assembly generated by Unicycler v0.4.6 (10) revealed the presence of a poxtA-like gene, named poxtA2, carried on a 13,746-bp plasmid, named pIB-BOL (GenBank accession number MZ171245) (Fig. 1a). The plasmid carried a repB gene closely related (98\% identity) to that of an 11-kb plasmid from a cfr-harboring Enterococcus faecalis of animal origin from China (GenBank accession number CP028840.1) and also a fexA phenicol resistance determinant, located upstream of poxtA2. Both genes were flanked by IS1216-like insertion sequences, likely involved with their mobilization (Fig. 1a).

Unlike poxtA, poxtA2 was not truncated by an IS1216 insertion at the $3^{\prime}$ end (Fig. 1b). Consequently, PoxtA2 differed from PoxtA by a few amino acids at the $\mathrm{C}$ terminus, which showed some detectable homology with the closest relative of the same protein family, namely, OptrA (Fig. 1c). Altogether, these findings suggest that poxtA2 likely represents the ancestor of poxtA, mobilized by a recombination event that had not truncated the gene at the $3^{\prime}$-end. In fact, the genetic context of poxtA2 was different from that of poxtA (Fig. 1b), supporting the notion that the two genes had been mobilized by independent recombination events.
Citation Baccani I, Antonelli A, Di Pilato V Coppi M, Di Maggio T, Spinicci M, Villagran AL, Revollo C, Bartoloni A, Rossolini GM. 2021 Detection of poxtA2, a presumptive poxtA ancestor, in a plasmid from a linezolid-resistant Enterococcus gallinarum isolate. Antimicrob Agents Chemother 65:e00695-21. https://doi .org/10.1128/AAC.00695-21

Copyright $\odot 2021$ American Society for Microbiology. All Rights Reserved. Address correspondence to Gian Maria Rossolini, gianmaria.rossolini@unifi.it. Accepted manuscript posted online 7 June 2021

Published 16 July 2021 
a

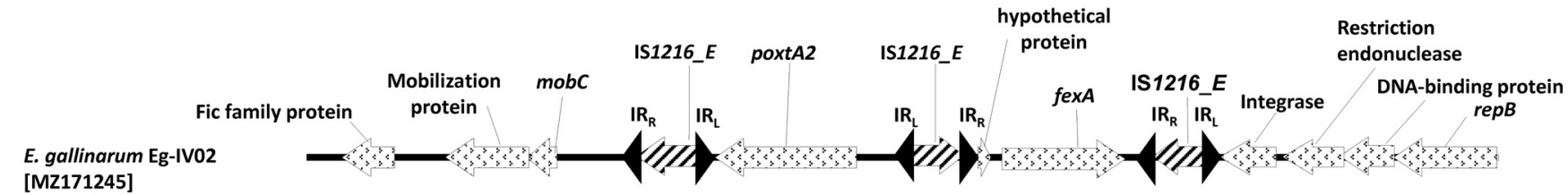

b

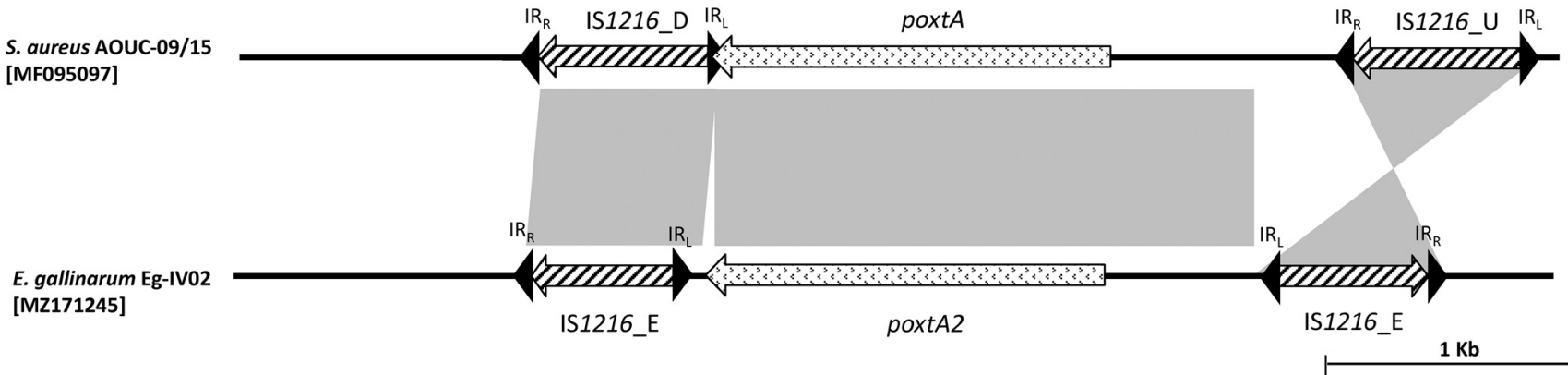

C

S. aureus AOUC-09/15

E. gallinarum Eg-IV02 [MZ171245]

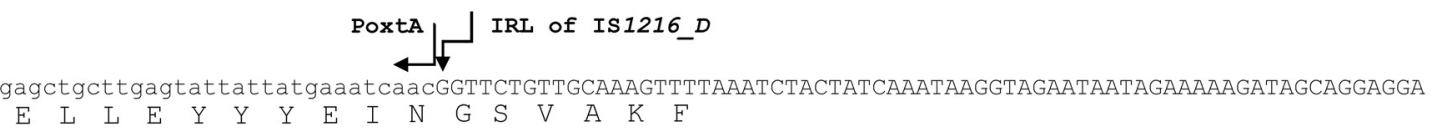

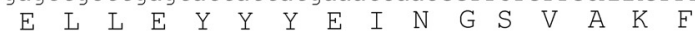

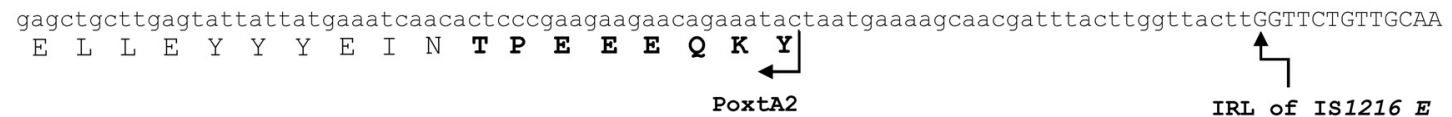

E. faecalis optrA [KP399637]

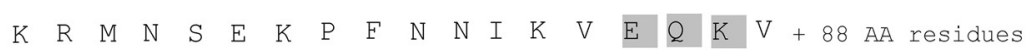

d E. gallinarum Eg-IV02
[MZ171245]

E. faecalis EFS0019

[NZ_QUSQ01000020.1]

E. gallinarum Eg-IV02 [MZ171245]

\section{E. faecalis EFS0019} [NZ_QUSQ01000020.1]

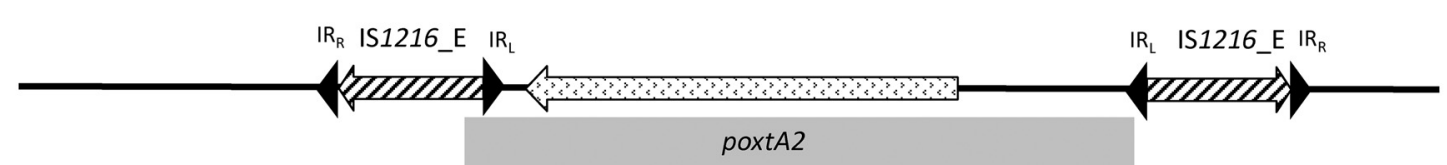

poxtA2

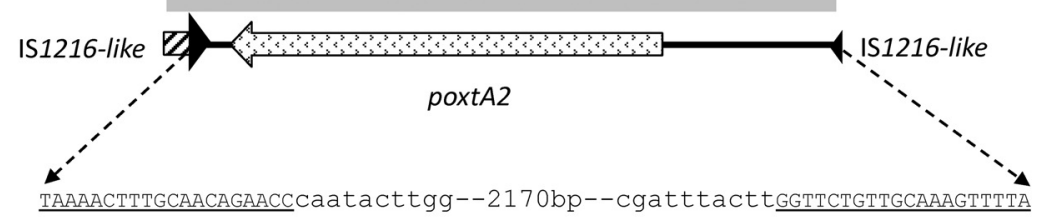

TAAAACTTTGCAACAGAACCCaatacttgg--2170bp--cgatt tact tGGTTCTGTTGCAAAGTTTTA

FIG 1 (a) Map of pIB-BOL plasmid from E. gallinarum Eg-IV02. (b) Comparison of the genetic context of poxtA in the chromosome of S. aureus AOUC-09/15 (1) and in plasmid pIB-BOL; regions with $>99 \%$ nucleotide identity are connected by gray zones, transposase-encoding genes are indicated by striped arrows, and IRs are indicated by black triangles. (c) Nucleotide sequences showing the insertion points of IS1216_D at the 3'-end of poxtA and of IS1216_E downstream poxtA2. The sequence of IS1216 elements is capitalized. Translation of the poxtA and poxtA2 coding sequences is also shown, and the different residues at the $C$ terminus of Poxt 2 are boldfaced. Alignment of the $C$ terminus of Poxt $A$ and Poxt 2 with the homologous region of OptrA is also shown. (d) Comparison of the genetic contexts of poxtA2 and of the identical gene recently reported from an E. faecalis EFS0019 of animal origin from Korea (GenBank accession number NZ_QUSQ01000020.1) (12). The gray shaded zones indicate 100\% nucleotide sequence homology. The sequences of the junctions are also shown (uppercase letters for IS1216 elements), revealing the identity between the two genetic contexts.

Plasmid pIB-BOL was transferred to E. faecalis $\mathrm{JH2}$-2 by electrotransformation (11). Transformants were selected on tryptic soy agar containing $8 \mu \mathrm{g} / \mathrm{ml}$ florfenicol. Acquisition of pIB-BOL by $E$. faecalis $\mathrm{JH} 2-2$ was associated with a significant increase of linezolid, florfenicol, and chloramphenicol MICs and with a lower increase of doxycycline MIC, while susceptibility to tedizolid, tetracycline, and tigecycline was apparently 
not affected (see Table S1 in the supplemental material). Since poxtA2 and fexA were the only resistance determinants carried by the plasmid (Fig. 1a), these results confirmed that poxtA2 was functional in conferring protection from linezolid, while the effect on phenicols could be ascribed to fexA.

A search of the NCBI sequence databases (carried out on 25 May 2021) revealed only another poxtA-like gene identical to poxtA2 from an $E$. faecalis isolate of animal origin from Korea (GenBank accession number NZ_QUSQ01000020.1) (12). Even that gene was flanked by two IS1216-like elements and exhibited a genetic context identical to that of poxtA2 from pIB-BOL (Fig. 1d), suggesting a common origin from a unique mobilization event. Interestingly, the same database search also revealed two poxtAlike genes encoding proteins identical to PoxtA but for a single amino acid change ( $\mathrm{G} 33 \mathrm{H}$ or $\mathrm{R} 256 \mathrm{H}$, respectively) from different Enterococcus faecium isolates in China (NCBI:protein accession numbers WP_212481470.1 and WP_159373727.1, respectively).

Overall, these findings identified a poxtA-related gene, which (i) appears to be the presumptive ancestor of poxt $A$ and (ii) has been mobilized to enterococci circulating in human and animal settings. The intersectoral spreading of these resistance determinants, mediating transferable linezolid resistance, is concerning and mandates for surveillance. In this perspective, it should be noted that the prevalence of poxtA2 might be underestimated in case of screening using primers targeting external regions of the poxtA gene.

Data availability. Sequence data have been deposited in GenBank under the accession number MZ171245.

\section{SUPPLEMENTAL MATERIAL}

Supplemental material is available online only.

SUPPLEMENTAL FILE 1, PDF file, $0.2 \mathrm{MB}$.

\section{ACKNOWLEDGMENT}

This study was supported by internal funding.

\section{REFERENCES}

1. Antonelli A, D'Andrea MM, Brenciani A, Galeotti $C L$, Morroni G, Pollini $S$, Varaldo PE, Rossolini GM. 2018. Characterization of poxtA, a novel phenicoloxazolidinone-tetracycline resistance gene from an MRSA of clinical origin. J Antimicrob Chemother 73:1763-1769. https://doi.org/10.1093/jac/dky088.

2. Fioriti S, Morroni G, Coccitto SN, Brenciani A, Antonelli A, Di PV, Baccani I, Pollini S, Cucco L, Morelli A, Paniccià M, Magistrali CF, Rossolini GM, Giovanetti E. 2020. Detection of oxazolidinone resistance genes and characterization of genetic environments in enterococci of swine origin, Italy. Microorganisms 8:2021. https://doi.org/10.3390/microorganisms8122021.

3. Na SH, Moon DC, Kim MH, Kang HY, Kim SJ, Choi JH, Mechesso A-F, Yoon S-S, Lim S-K. 2020. Detection of the phenicol-oxazolidinone resistance gene poxtA in Enterococcus faecium and Enterococcus faecalis from foodproducing animals during 2008-2018 in Korea. Microorganisms 8:1839. https://doi.org/10.3390/microorganisms8111839.

4. Li D, Cheng Y, Schwarz S, Yang M, Du X-D. 2019. Identification of a poxtAand cfr-carrying multiresistant Enterococcus hirae strain. J Antimicrob Chemother 75:482-484.

5. Papagiannitsis CC, Tsilipounidaki K, Malli E, Petinaki E. 2019. Detection in Greece of a clinical Enterococcus faecium isolate carrying the novel oxazolidinone resistance gene poxtA. J Antimicrob Chemother 74:2461-2462. https://doi.org/10.1093/jac/dkz155.

6. Freitas AR, Tedim AP, Duarte B, Elghaieb H, Abbassi MS, Hassen A, Read A, Alves V, Novais C, Peixe L. 2020. Linezolid-resistant (Tn6246::fexB-poxtA) Enterococcus faecium strains colonizing humans and bovines on different continents: similarity without epidemiological link. J Antimicrob Chemother 75:2416-2423. https://doi.org/10.1093/jac/dkaa227.

7. Egan SA, Shore AC, O'Connell B, Brennan Gl, Coleman DC. 2020. Linezolid resistance in Enterococcus faecium and Enterococcus faecalis from hospitalized patients in Ireland: high prevalence of the MDR genes optrA and poxtA in isolates with diverse genetic backgrounds. J Antimicrob Chemother 75: 1704-1711. https://doi.org/10.1093/jac/dkaa075.

8. Moure Z, Lara N, Marín M, Sola-Campoy PJ, Bautista V, Gómez-Bertomeu F, Gómez-Dominguez C, Pérez-Vázquez M, Aracil B, Campos J, Cercenado E, Oteo-Iglesias J, Spanish Linezolid-Resistant Enterococci Collaborating Group. 2020. Interregional spread in Spain of linezolid-resistant Enterococcus spp. isolates carrying the optrA and poxtA genes. Int J Antimicrob Agents 55:105977. https://doi.org/10.1016/j.ijantimicag.2020.105977.

9. International Organization for Standardization. 2019. ISO 20776-1: Clinical laboratory testing and in vitro diagnostic test systems. Susceptibility testing of infectious agents and evaluation of performance of antimicrobial susceptibility test devices-Part 1: Reference method for the in vitro activity of antimicrobial agents against rapidly growing aerobic bacteria involved in infectious diseases. International Organization for Standardization, Geneva, Switzerland.

10. Coppi M, Di Pilato V, Monaco F, Giani T, Conaldi PG, Rossolini GM. 2020. Ceftazidime-avibactam resistance associated with increased $b / a_{\mathrm{KPC}-3}$ gene copy number mediated by $\mathrm{pKpQIL}$ plasmid derivatives in sequence type 258 Klebsiella pneumoniae. Antimicrob Agents Chemother 64:e01816-19. https://doi.org/10.1128/AAC.01816-19.

11. Brenciani A, Morroni G, Pollini S, Tiberi E, Mingoia M, Varaldo PE, Rossolini GM, Giovanetti E. 2016. Characterization of novel conjugative multiresistance plasmids carrying cfr from linezolid-resistant Staphylococcus epidermidis clinical isolates from Italy. J Antimicrob Chemother 71:307-313. https://doi.org/ 10.1093/jac/dkv341.

12. Jung YH, Cha MH, Woo GJ, Chi YM. 2021. Characterization of oxazolidinone and phenicol resistance genes in non-clinical enterococcal isolates from Korea. J Glob Antimicrob Resist 24:363-369. https://doi.org/10.1016/j.jgar.2021.01.009. 